\title{
Salivary Metabolomics Fingerprint of Chronic Apical Abscess with Sinus Tract: A Pilot Study
}

\author{
Noemi Montis, ${ }^{1}$ Elisabetta Cotti, ${ }^{1}$ Antonio Noto $\left(\mathbb{D},{ }^{2}\right.$ Claudia Fattuoni $\left(\mathbb{D},{ }^{3}\right.$ \\ and Luigi Barberini $\mathbb{B}^{2}$ \\ ${ }^{1}$ Department of Conservative Dentistry and Endodontics, University of Cagliari, Cagliari, Italy \\ ${ }^{2}$ Department of Medical Sciences and Public Health, University of Cagliari, Cagliari, Italy \\ ${ }^{3}$ Department of Chemical and Geological Sciences, University of Cagliari, Cagliari, Italy \\ Correspondence should be addressed to Claudia Fattuoni; cfattuon@unica.it
}

Received 26 September 2019; Accepted 26 October 2019; Published 16 November 2019

Academic Editor: Meehir Palit

Copyright (c) 2019 Noemi Montis et al. This is an open access article distributed under the Creative Commons Attribution License, which permits unrestricted use, distribution, and reproduction in any medium, provided the original work is properly cited.

Chronic apical abscess (CAA) is a lesion of apical periodontitis mostly characterized by areas of liquefactive necrosis with disintegrating polymorphonuclear neutrophils surrounded by macrophages. Its presence leads to local bacterial infection, systemic inflammatory response, pain, and swelling. The use of a novel approach for the study of CAA, such as metabolomics, seems to be important since it has proved to be a powerful tool for biomarkers discovery which could give novel molecular insight on CAA. So, the aim of this study was to verify the possibility to identify the metabolic fingerprint of CAA through the analysis of saliva samples. Nineteen patients were selected for this study: eleven patients affected by CAA with a sinus tract constituted the study group whereas eight patients without clinical and radiographic signs of CAA formed the healthy control group. Saliva samples were collected from each subject and immediately frozen at $-80^{\circ} \mathrm{C}$. Metabolomic profiles were obtained using a gas chromatography/mass spectrometry instrument. Subsequently, in order to compare the two groups, a multivariate statistical model was built that resulted to be statistically significant. The class of metabolites characterizing the CAA patients was closely related to the bacterial catabolism, tissue necrosis, and presence of a sinus tract. These preliminary results, for the first time, indicate that saliva samples analyzed by means of GC/MS metabolomics may be useful for identifying the presence of CAA, leading to new insights into this disease.

\section{Introduction}

Apical periodontitis (AP) is a pathologic condition of the oral periradicular tissues generated as a consequence of the inflammatory response at the root apices of teeth with necrotic infected pulps. Endodontic infections are usually restricted to the root canal system and include microorganisms, viruses, fungi, and yeasts [1-4]. The inflammatory response is believed to be an attempt to prevent the spread of the infection to the bone and to the entire system [5]. However, in some cases, microorganisms from the root canal may extend to the periradicular tissues and cause an extraradicular infection usually associated with symptoms and/or persistent apical periodontitis [6,7]. Chronic apical abscess (CAA) is a lesion of AP which is mostly characterized by areas of liquefactive necrosis with disintegrating polymorphonuclear neutrophils (PMNs) surrounded by macrophages and normal PMNs [8]. This condition is associated with the intermittent drainage of the purulent content via a sinus tract, which opens in the oral mucosa (intraoral) or, less frequently, in the skin (extraoral) [5,6,9-11]. Sinus tracts in most cases exhibit an epithelial lining at the stoma, while its pathway may show an epithelial coating or be lined by an inflamed connective tissue $[11,12]$. CAA has a prevalence of $8.5 \%-18 \%$, in AP it is usually asymptomatic, often associated with larger, long-standing lesions ( $>5 \mathrm{~mm}$ in diameter) and an extraradicular infection $[1,7,12-16]$. Interestingly, the presence of a CAA with a sinus tract is considered a negative prognostic factor in the outcome of primary and secondary endodontic treatments; 
therefore, the use of novel approaches for the study of CAA seems important.

Metabolomics is one of the most recent research technologies focused on the quantitative and qualitative analyses of low-molecular weight metabolites (less than 1500 Daltons) present in biological fluids in response to a pathophysiological status [17]. It creates an instant snapshot of the biological status of a given organism, representing a sensitive measure of the biological status in health or disease [18]. The identification of an altered metabolic fingerprints offers novel opportunities to better understand physiological state, detect or identify potential risks for various diseases, and ultimately help achieve the goal of "personalized medicine." It has been recently hypothesized that the presence of CAA can permanently modify the saliva metabolic content; therefore, it represents an important area for the aforementioned interventions [19]. This study aimed to identify the metabolic fingerprint of CAA patients through the metabolomic analysis of saliva and to open a novel insight into chronic apical periodontitis.

\section{Materials and Methods}

2.1. Clinical Population. This study was conducted on men and women observed at the Dental Clinic of the University of Cagliari, Italy, and was approved by the ethics committee. Following the recording of the medical and dental history, all patients received an accurate dental evaluation. A panoramic radiograph was performed as initial screening followed by intraoral radiographs taken on selected areas. Clinical examination was then performed on each individual and included the evaluation of hard and soft tissues (the presence and location of swelling and sinus tracts), confirming diagnosis of CAA by following sensitivity tests, cold test, palpation, percussion, and periodontal probing. Intraoral periapical radiographs were taken on all suspect teeth and all teeth were affected by CAA, tracing the sinus tract with a gutta-percha cone. The patients were then assigned to two groups according to the inclusion and exclusion criteria. Inclusion criteria were as follows: the presence of at least one tooth affected by CAA with a sinus tract, as assessed clinically and radiographically; good oral hygiene; presence of at least 20 teeth; absence of signs and symptoms of periodontal disease; absence of any known systemic disease; absence of pharmacological therapy; a range between 30 and 60 years of age. Exclusion criteria were as follows: the presence of poor oral hygiene; presence of localized or diffuse, chronic, or aggressive periodontitis; presence of destroying caries; patients who underwent chemotherapy or radiant therapy; patients who have been subjected to surgical dental procedures less than 15 days earlier (extraction, implant, periodontal, or endodontic therapies); patients under orthodontic therapy.

Nineteen subjects were divided into two groups. All subjects signed a written informed consent. Eleven patients affected by CAA formed the study group (group 1, average: 47 years old; standard deviation: 11.7 years old), while 8 patients free from clinical and radiographic evidence of CAA or AP were the healthy controls (group 2, average: 43.2 years
TABle 1: Clinical and demographic characteristics of patients population.

\begin{tabular}{lccc}
\hline Patient ID & Gender & Age (years) & Histopathologic diagnosis \\
\hline 1 & Female & 30 & CAA \\
2 & Male & 51 & CAA \\
3 & Male & 33 & CAA \\
4 & Female & 60 & CAA \\
5 & Male & 42 & CAA \\
6 & Female & 68 & CAA \\
7 & Male & 43 & CAA \\
8 & Male & 39 & CAA \\
9 & Male & 60 & CAA \\
10 & Male & 46 & CAA \\
11 & Male & 45 & CAA \\
12 & Male & 37 & Control \\
13 & Male & 30 & Control \\
14 & Male & 41 & Control \\
15 & Male & 38 & Control \\
16 & Female & 39 & Control \\
17 & Female & 45 & Control \\
18 & Female & 56 & Control \\
19 & Female & 60 &
\end{tabular}

old; standard deviation: 10.8 years old). Patients had similar demographic data (race, sex, and age distribution) (Table 1).

2.2. Metabolomic Analysis. Saliva samples (2 mL) were collected from each subject and immediately frozen at $-80^{\circ} \mathrm{C}$. The samples were subsequently thawed at room temperature, and $1 \mathrm{~mL}$ of saliva was transferred in an Eppendorf tube with $1 \mathrm{~mL}$ of cold acetone $\left(-20^{\circ} \mathrm{C}\right)$, vortexed for $30 \mathrm{~s}$, and centrifuged $\left(14000 \mathrm{rpm}\right.$ at $4^{\circ} \mathrm{C}$ for $\left.10 \mathrm{~min}\right)$ to remove the proteins [20]. $1 \mathrm{~mL}$ of supernatant was then transferred into glass vials and evaporated to dryness overnight in an Eppendorf vacuum centrifuge (Eppendorf AG, Hamburg, Germany). $30 \mu \mathrm{L}$ of a $0.24 \mathrm{M}$ solution of methoxylamine hydrochloride in pyridine was added to each vial, and samples were vortex mixed and left to react for $17 \mathrm{~h}$ at room temperature. In the following step, $30 \mu \mathrm{L}$ of MSTFA ( $N$-methyl- $N$-trimethylsilyltrifluoroacetamide) was added to the vials and left to react for $1 \mathrm{~h}$ at room temperature. Finally, the derivatized samples were diluted with hexane $(600 \mu \mathrm{L})$ and tetracosane $(0.01 \mathrm{mg} / \mathrm{ml})$ as internal standard, just before GC-MS analysis. Samples were analyzed using an Agilent 5977B interfaced to the GC 7890B (Agilent Technologies, Palo Alto, CA, USA), equipped with a DB-5ms column (Agilent J \& W Scientific, Folsom, CA, USA), injector temperature at $230^{\circ} \mathrm{C}$, detector temperature at $280^{\circ} \mathrm{C}$, and helium carrier gas flow rate of $1 \mathrm{~mL} / \mathrm{min}$. The GC oven temperature program was set at $90^{\circ} \mathrm{C}$ initial temperature with $1 \mathrm{~min}$ hold time and ramping at $10^{\circ} \mathrm{C} / \mathrm{min}$ to a final temperature of $270^{\circ} \mathrm{C}$, with $7 \mathrm{~min}$ hold time. $1 \mu \mathrm{L}$ of the derivatized sample was injected in the split $(1: 20)$ mode. After a solvent delay of 3 minutes, mass spectra were acquired in full scan mode, using 2.28 scans/s with a mass range of 50-700 amu.

All acquired chromatograms were analyzed using the free software AMDIS (Automated Mass Spectral 
Deconvolution and Identification System, http://chemdata. nist.gov/mass-spc/amdis). Each chromatographic peak was identified by comparing the relative mass spectrum and retention time with those stored in an in-house made library including 234 metabolites. Other metabolites were identified using NIST08 (the National Institute of Standards and Technology's mass spectral database) and Golm Metabolome Database (GMD, http://gmd.mpimp-golm.mpg.de/). The metabolite was considered positively identified with a match factor $\geq 70 \%$.

This strategy allowed for the detection of 76 compounds: 70 accurately identified and 6 unknown molecules recurring in every sample. AMDIS analysis produced an electronic sheet data matrix (Microsoft ${ }^{\circledR}$ Excel $^{\circledR}$, Microsoft Co, Redmond Washington DC, USA) that was submitted to multivariate statistical analysis. Data matrix was analyzed by the multivariate statistical analysis method, called orthogonal partial least square discriminant analysis (OPLS$\mathrm{DA}$ ), to obtain the set of important variables (metabolites) between the groups (SIMCA-P+ program, Version 13.0, Umetrics, Umea, Sweden). Subsequently, as metabolites are associated with biological functions, a pathway analysis was performed to discover a functional interpretation. The resulting "metabolome view" depicted all metabolic pathways arranged according to the scores from the enrichment analysis ( $y$-axis) and from topology analysis ( $x$ axis). Since many pathways were tested simultaneously, $p$ values were adjusted for multiple testing. The goal of the pathway analysis was to identify the pathways which have a significant impact in a given phenotype and to verify whether the most important pathway modification resulted was affected by the periapical lesions and the sinus tract [21].

\section{Results}

Eleven CAA cases (green dots) and 8 healthy controls (blue dots) underwent metabolomics analysis. The multivariate statistical analysis showed a clear separation between cases and controls. This model was best described by the two principal components, showing $R^{2}$ (goodness of fit) $=0.793$ and $\mathrm{Q}^{2}$ (goodness of prediction) $=0.358 ; p$ value $<0.05$ (Figures 1(a) and 1(b)).

Among the 76 metabolites detected, the CAA group exhibited statistically higher concentrations of 4-hydroxyhydrocinnamic acid, $\mathrm{N}$-acetylneuraminic acid, inositollike (an inositol isomer other than myo-, scyllo-, or chiroinositol), ornithine, putrescine, hypoxanthine, 5-aminopentanoic acid, proline, uracil, lysine, stearic acid, threonine, uric acid, glycine, and phosphoethanolamine with respect to the control group. On the other hand, the CAA group showed statistically lower concentrations of sorbitol, maltose, glucose, xylitol, succinic acid, ethanolamine, lactic acid, palmitic acid, citric acid, urea, 1,2propanediol, and meso-2,3-butanediol when compared to the controls (Table 2).

The pathway analysis allowed the identification of four distinctive pathways among the CAA patients. The resulted pathways were purine nucleotide cycle, amino sugar and nucleotide sugar metabolism, pentose phosphate pathway, and glycolysis and gluconeogenesis pathways (Table 3).

\section{Discussion}

Chronic apical abscess is an inflammatory disease affecting the periapical tissues and triggered by root canal infection. Among the factors contributing to the development of CAA, a shift in the homeostasis of the oral bacterial towards the dysbiosis of the microbial population is considered important [22]. In this respect, metabolomics enables studies of substances that may turn out to be candidate biomarkers of such condition. In fact, discriminant metabolites constituting the fingerprint of the disease may help to better understand the features sustaining a chronic endodontic infection and to establish better strategies to prevent and deal with these conditions. Recent studies in the fields of metabolomics provided novel information on the pathogenesis of periodontal inflammation, infection, and tissue destruction. All these studies were performed using the saliva, as the biofluid of choice, due to its close contact with the overall oral cavity [23]. Moreover, saliva is easier to handle than blood because it does not clot, thus reducing the number of manipulations required. Saliva is composed of water for $99 \%$ of its volume, and the remaining $1 \%$ comprises hundreds of small molecules that make it an interesting biofluid for medical testing. The chemical composition of saliva is known to change dramatically in response to different physiological and pathological stimuli [24]. The salivary metabolome of CAA patients in this study presented heavy differences compared to the healthy population, as evidenced by the metabolite list reported in Table 2. The most important biochemical pathways that characterized the saliva from CAA patients were as follows: pentose-phosphate pathways, amino sugar and nucleotide metabolism, glycolysis or gluconeogenesis, and purine nucleotide cycle. Among the metabolites from the abovementioned pathways, some reflect the differences between the two groups of patients. In particular, high levels of 4hydroxyhydrocinnamic, putrescine, ornithine, hypoxanthine, and phosphoethanolamine and lower levels of ethanolamine and uric acid have already been associated with periodontal inflammatory status in a recent metabolomic study performed in adult patients before and after the removal of supragingival plaque [25]. The authors were eventually able to build a ROC curve by using 4-hydroxyhydrocinnamic and cadaverine that resulted to be highly specific for the identification of severe periodontitis. Furthermore, the production of 4-hydroxyhydrocinnamic was hypothesized to be related to the gut microbiota dysbiosis [26].

Putrescine is a polyamine synthesized by bacteria, such as E. coli and Pseudomonas species, via two pathways, the decarboxylation of ornithine to putrescine and that of arginine to agmatine, followed by the conversion of agmatine to putrescine and urea [27]. The presence of high level of putrescine could be intended as an adaptation mechanism of the bacteria to counteract a low $\mathrm{pH}$ to induce the alkalinisation of the cytosol and the generation of a proton motive 


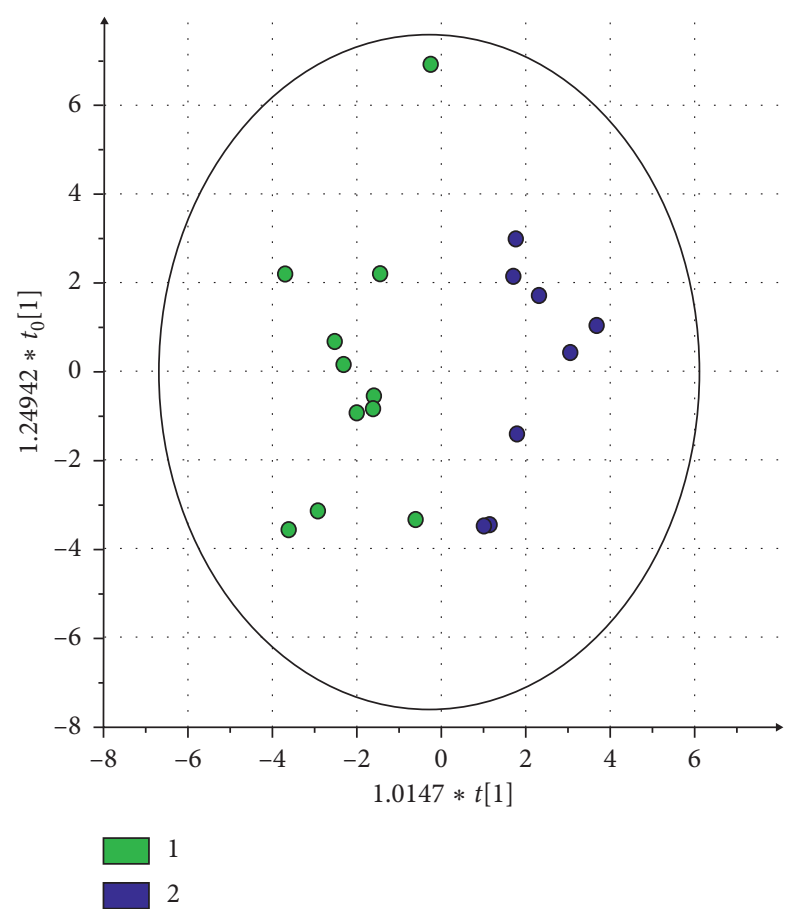

(a)

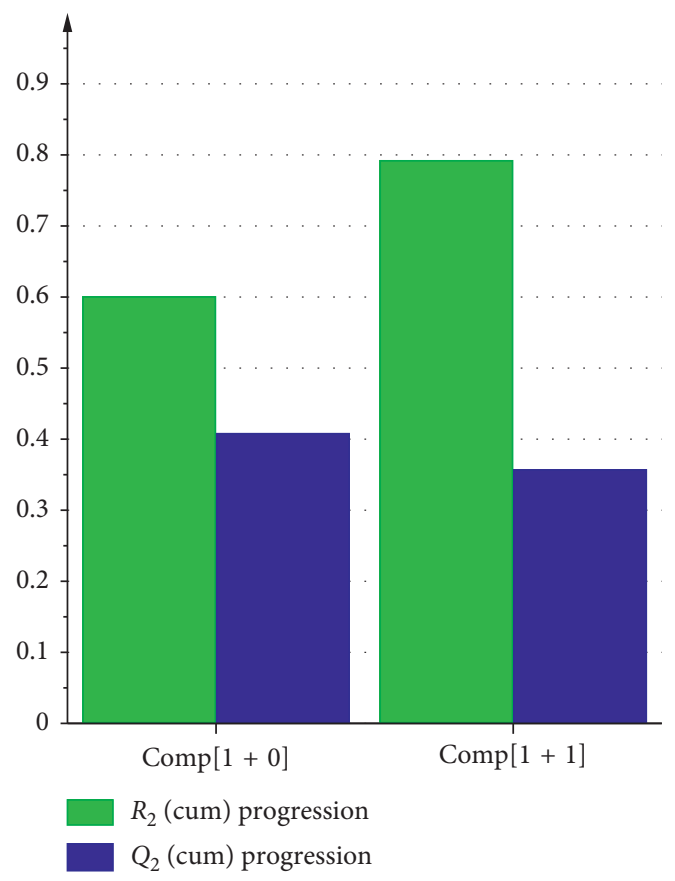

(b)

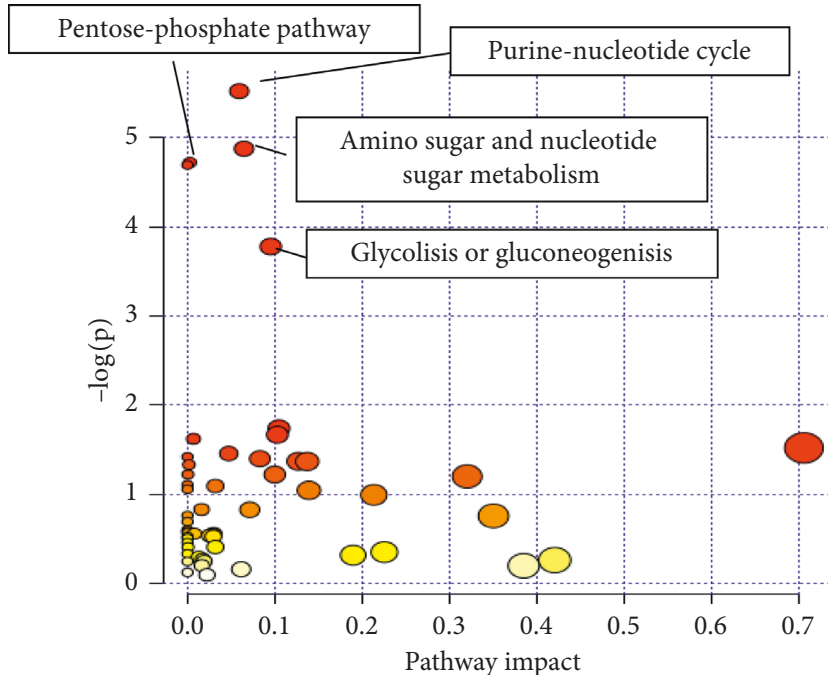

(c)

Figure 1: (a) The OPLS-DA model indicated a clear separation between the groups; (b) cross validation $\left(R^{2}=0.793 Q^{2}=0.358\right)$; $(\mathrm{c})$ metabolic hubs mainly affected in CAA patients.

force useful for acid stress resistance and for ATP production [28].

The bacterial metabolic fingerprint was also indicated by the presence of a high concentration of phosphoethanolamine, a phosphomonoester metabolite of the phospholipid metabolism, precursor of phospholipid synthesis, and product of phospholipid breakdown. Lipids seem to have an important role in the oral ecosystem and their concentration correlates with the presence of caries [29]. Of interest, reduced levels of ethanolamine by-product in CAA patients has been related to bacterial dysbiosis, since this compound is readily derived from cell membranes that certain bacteria can utilize as a carbon/nitrogen source [30].

Another metabolite related to bacterial overgrowth and/ or endogenous tissue necrosis is 5-aminopentanoic acid, whose high concentration is known to be present in patients with chronic periodontitis [31]. Its metabolism seems to be indicative of the activity of anaerobic bacteria such as Clostridium viridans [32].

High concentrations of hypoxanthine and uric acid, apparently in contrast, seem to be related to the oxidative stress: hypoxanthine indicates the activation of the purine degradation pathway, a major biochemical source to 
TABLE 2: Variable influence in projection (metabolites accumulated or depleted in CAA patients sorted by trend).

\begin{tabular}{|c|c|c|}
\hline \multicolumn{3}{|c|}{ VIP: variable influence in projection } \\
\hline Metabolites & Trend in CAA patients & Score values sorted by trend \\
\hline 4-Hydroxyhydrocinnamic acid & $\uparrow$ & 1.90102 \\
\hline$N$-Acetylneuraminic acid & $\uparrow$ & 1.70824 \\
\hline Inositol-like & $\uparrow$ & 1.35246 \\
\hline Ornithine & $\uparrow$ & 1.2671 \\
\hline Putrescine & $\uparrow$ & 1.25158 \\
\hline Phosphoethanolamine & $\uparrow$ & 1.1257 \\
\hline Hypoxanthine & $\uparrow$ & 1.07426 \\
\hline 5-Aminopentanoic acid & $\uparrow$ & 0.99078 \\
\hline Proline & $\uparrow$ & 0.948403 \\
\hline Uracil & $\uparrow$ & 0.804598 \\
\hline Lysine & $\uparrow$ & 0.764083 \\
\hline Stearic acid & $\uparrow$ & 0.698099 \\
\hline Threonine & $\uparrow$ & 0.654658 \\
\hline Uric acid & $\uparrow$ & 0.630694 \\
\hline Glycine & $\uparrow$ & 0.620945 \\
\hline Sorbitol & $\downarrow$ & 4.42798 \\
\hline Maltose & $\downarrow$ & 3.66992 \\
\hline Glucose & $\downarrow$ & 2.58188 \\
\hline Xylitol & $\downarrow$ & 1.74627 \\
\hline Succinic acid & $\downarrow$ & 1.26194 \\
\hline Ethanolamine & $\downarrow$ & 1.17785 \\
\hline Lactic acid & $\downarrow$ & 1.13476 \\
\hline Palmitic acid & $\downarrow$ & 1.02905 \\
\hline Citric acid & $\downarrow$ & 0.995201 \\
\hline Urea & $\downarrow$ & 0.886784 \\
\hline 1,2-Propanediol & $\downarrow$ & 0.774375 \\
\hline meso-2,3-butanediol & $\downarrow$ & 0.67866 \\
\hline
\end{tabular}

TABLE 3: Most important pathways based on the metabolic fingerprint.

\begin{tabular}{lccc}
\hline & \multicolumn{2}{c}{ Pathways mainly affected in CAA patients } \\
\hline Pathway name & $p$ value & False discovery rate (FDR) & $\begin{array}{c}\text { Metabolites belonging to the pathway } \\
\text { Hypoxanthine, urea, uric acid, glycine, inositol, } \\
\text { urea, 1,2-propanediol }\end{array}$ \\
Purine nucleotide cycle & 0,004 & 0,125 & $\begin{array}{c}\text { Glucose, pyruvic acid, } N \text {-acetylneuraminic acid } \\
\text { Amino sugar and nucleotide sugar metabolism }\end{array}$ \\
$\begin{array}{l}\text { Pentose phosphate pathway } \\
\text { Glycolysis and gluconeogenesis pathways }\end{array}$ & 0,009 & 0,125 & $\begin{array}{c}\text { Glucose, pyruvic acid, phosphate, ethanolamine } \\
\text { Lactic acid, pyruvic acid, glucose, citric acid, } \\
\text { succinic acid }\end{array}$ \\
\hline
\end{tabular}

produce reactive oxygen species (ROS) [33]. High concentration of uric acid, a well-known salivary antioxidant could on the other hand be interpreted as a response of the organism to the ROS production. Redox balance is crucial for the maintenance of normal cellular function and health. The reduction of antioxidants in periodontal diseases has been well documented in the literature [34]. Furthermore, an indirect link with the purine degradation pathway could be hypothesized through the detected decrement of succinate, a Krebs cycle intermediate. The metabolic connection between the purine pathway and the Krebs cycle via the "purine nucleotide cycle" is well known in the biological literature.

\section{Conclusion}

The results of our study should be cautiously interpreted due to the following limitations. The size of our model was too small to draw general extrapolations on the pathophysiological events underlying this complex clinical scenario. The limited number of subjects was due to the need to have all the patients treated in the same way by the same physician in order to reduce all clinical and pharmacological biases. For this reason, all biological samples were collected in a single Dental Clinic at the University Hospital. Nonetheless, the interesting features of this pilot study are the metabolites identified in CAA patients, which seem to be closely related to bacterial catabolism and tissue necrosis, which in turn may be associated with the presence of a sinus tract.

\section{Data Availability}

The data used to support the findings of this study are available from the corresponding author upon request.

\section{Conflicts of Interest}

The authors declare no conflicts of interest regarding the publication of this study. 


\section{References}

[1] D. Ricucci and J. F. Siqueira Jr, "Biofilms and apical periodontitis: study of prevalence and association with clinical and histopathologic findings," Journal of Endodontics, vol. 36, no. 8, pp. 1277-1288, 2010.

[2] P. V. Abbott, "Classification, diagnosis and clinical manifestations of apical periodontitis," Endodontic Topics, vol. 8, no. 1, pp. 36-54, 2004.

[3] J. Slots, "Herpesviruses in periodontal diseases," Periodontology 2000, vol. 38, no. 1, pp. 33-62, 2005.

[4] A. Contreras, M. Umeda, C. Chen, I. Bakker, J. L. Morrison, and J. Slots, "Relationship between herpesviruses and adult periodontitis and periodontopathic bacteria," Journal of Periodontology, vol. 70, no. 5, pp. 478-484, 1999.

[5] J. I. Ingle, L. K. Bakland, and J. C. Baumgartner, Ingle's Endodontics 6, Vol. 13, PMPH-USA, Raleigh, NC, USA, 2008.

[6] P. T. Sunde, I. Olsen, P. O. Lind, and L. Tronstad, "Extraradicular infection: a methodological study," Dental Traumatology, vol. 16, no. 2, pp. 84-90, 2000.

[7] M. Haapasalo, K. Ranta, and H. Ranta, "Mixed anaerobic periapical infection with sinus tract," Dental Traumatology, vol. 3, no. 2, pp. 83-85, 1987.

[8] J. F. Siqueira Jr, Treatment of Endodontic Infections, Quintessence Publishing, London, UK, 2011.

[9] American Association of Endodontists, Glossary of Endodontic Terms, AAE, Chicago, IL, USA, Ninth edition, 2016.

[10] K. M. Hargreaves and L. H. Berman, Cohen's Pathways of the Pulp Expert Consult, Elsevier Health Sciences, St. Louis, MO, USA, Eleventh edition, 2016.

[11] J. W. Harrison and W. J. Larson, "The epithelized oral sinus tract," Oral Surgery, Oral Medicine, Oral Pathology, vol. 42, no. 4, pp. 511-517, 1976.

[12] J. C. Baumgartner, A. B. Picket, and J. T. Muller, "Microscopic examination of oral sinus tracts and their associated periapical lesions," Journal of Endodontics, vol. 10, no. 4, pp. 146-152, 1984.

[13] H. Mortensen, J. E. Winther, and H. Birn, "Periapical granulomas and cysts," European Journal of Oral Sciences, vol. 78, no. 1-4, pp. 241-250, 1970.

[14] R. Gupta and G. Hasselgren, "Prevalence of odontogenic sinus tracts in patients referred for endodontic therapy," Journal of Endodontics, vol. 29, no. 12, pp. 798-800, 2003.

[15] J. Valderhaug, "A histologic study of experimentally produced intra-oral odontogenic fistulae in monkeys," International Journal of Oral Surgery, vol. 2, no. 2, pp. 54-61, 1973.

[16] R. Weiger, B. Manncke, H. Werner, and C. Lost, "Microbial flora of sinus tracts and root canals of non-vital teeth," Dental Traumatology, vol. 11, no. 1, pp. 15-19, 1995.

[17] J. K. Nicholson, J. C. Lindon, and E. Holmes, “"Metabonomics": understanding the metabolic responses of living systems to pathophysiological stimuli via multivariate statistical analysis of biological NMR spectroscopic data," Xenobiotica, vol. 29, no. 11, pp. 1181-1189, 1999.

[18] A. Klupczyńska, P. Dereziński, and Z. J. Kokot, "Metabolomics in medical sciences-trends, challenges and perspectives," Acta Poloniae Pharmaceutica, vol. 72, no. 4, pp. 629-641, 2015.

[19] N. Montis, C. Fattuoni, L. Barberini, A. Noto, V. Fanos, and E. Cotti, "Metabolomics fingerprint of chronic apical periodontitis with sinus tract: a pilot study," International Endodontic Journal, vol. 50, no. 1, pp. 3-56, 2017.

[20] J. Zheng, R. A. Dixon, and L. Li, "Development of isotope labeling LC-MS for human salivary metabolomics and application to profiling metabolome changes associated with mild cognitive impairment," Analytical Chemistry, vol. 84, no. 24, pp. 10802-10811, 2012.

[21] D. S. Wishart, Y. D. Feunang, A. Marcu et al., "HMDB 4.0: the human metabolome database for 2018," Nucleic Acids Research, vol. 46, no. D1, pp. D608-D617, 2018.

[22] S. Nath and R. Raveendran, "Microbial dysbiosis in periodontitis," Journal of Indian Society of Periodontology, vol. 17, no. 4, pp. 543-545, 2013.

[23] A. Wang, C. Wang, M. Tu, and D. Wong, "Oral biofluid biomarker research: current status and emerging frontiers," Diagnostics, vol. 6, no. 4, p. 45, 2016.

[24] Z. T. Dame, F. Aziat, R. Mandal et al., "The human saliva metabolome," Metabolomics, vol. 11, no. 6, pp. 1864-1883, 2015.

[25] A. Sakanaka, M. Kuboniwa, E. Hashino, T. Bamba, E. Fukusaki, and A. Amano, "Distinct signatures of dental plaque metabolic byproducts dictated by periodontal inflammatory status," Scientific Reports, vol. 7, no. 1, p. 42818, 2017.

[26] K. Ou, P. Sarnoski, K. R. Schneider, K. Song, C. Khoo, and L. $\mathrm{Gu}$, "Microbial catabolism of procyanidins by human gut microbiota," Molecular Nutrition \& Food Research, vol. 58, no. 11, pp. 2196-2205, 2014.

[27] S. S. Cohen, A Guide to the Polyamines, Oxford University Press, New York, NY, USA, 1997.

[28] D. Molenaar, J. S. Bosscher, B. T. Brink, A. J. Driessen, and W. N. Konings, "Generation of a proton motive force by histidine decarboxylation and electrogenic histidine/histamine antiport in Lactobacillus buchneri," Journal of Bacteriology, vol. 175, no. 10, pp. 2864-2870, 1993.

[29] Y. Tomita, N. Miyake, and S. Yamanaka, "Lipids in human parotid saliva with regard to caries experience," Journal of Oleo Science, vol. 57, no. 2, pp. 115-121, 2008.

[30] D. A. Garsin, "Ethanolamine utilization in bacterial pathogens: roles and regulation," Nature Reviews Microbiology, vol. 8, no. 4, pp. 290-295, 2010.

[31] S. Syrjänen, P. Piironen, and H. Markkanen, "Free amino-acid content of wax-stimulated human whole saliva as related to periodontal disease," Archives of Oral Biology, vol. 32, no. 9, pp. 607-610, 1987.

[32] W. Buckel, P. H. Janssen, A. Schuhmann et al., "Clostridium viride sp. nov., a strictly anaerobic bacterium using 5-aminovalerate as growth substrate, previously assigned to Clostridium aminovalericum," Archives of Microbiology, vol. 162, no. 6, pp. 387-394, 1994.

[33] V. M. Barnes, R. Teles, H. M. Trivedi et al., "Acceleration of purine degradation by periodontal diseases," Journal of Dental Research, vol. 88, no. 9, pp. 851-855, 2009.

[34] I. L. C. Chapple, G. Brock, C. Eftimiadi, and J. B. Matthews, "Glutathione in gingival crevicular fluid and its relation to local antioxidant capacity in periodontal health and disease," Molecular Pathology, vol. 55, no. 6, pp. 367-373, 2002. 


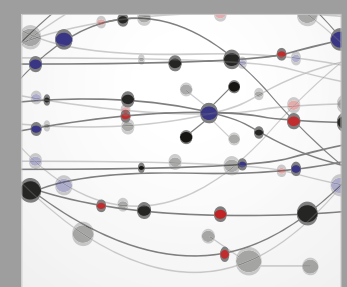

The Scientific World Journal
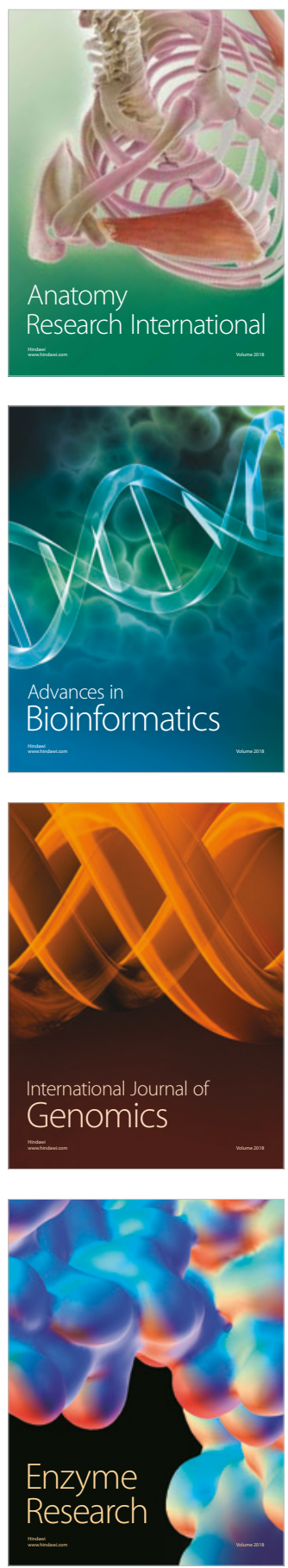
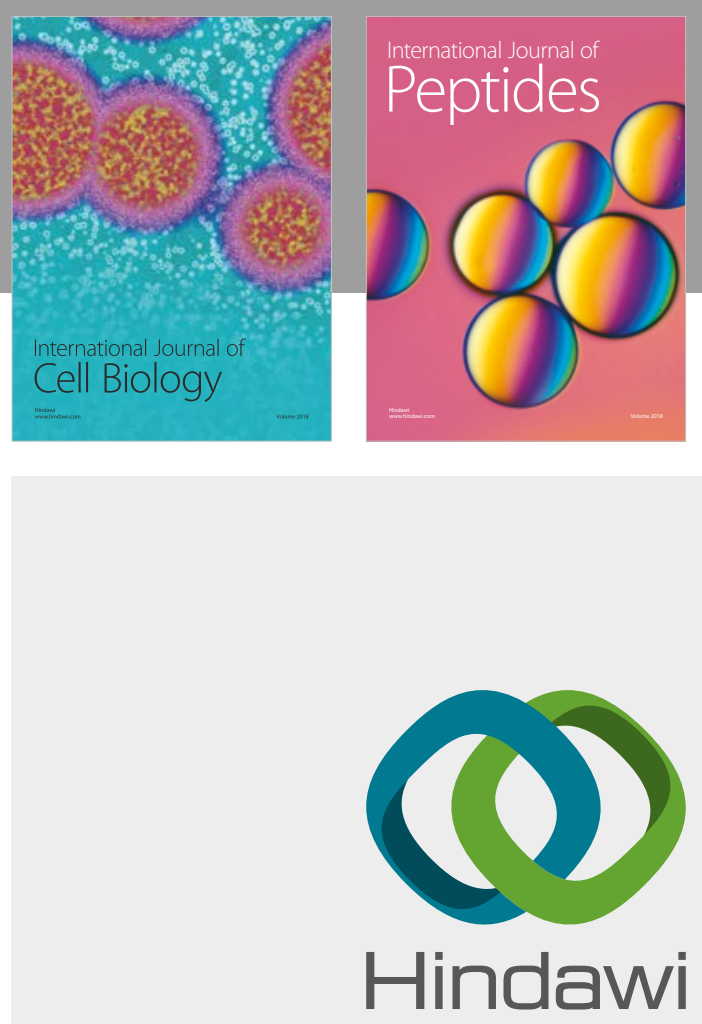

Submit your manuscripts at

www.hindawi.com
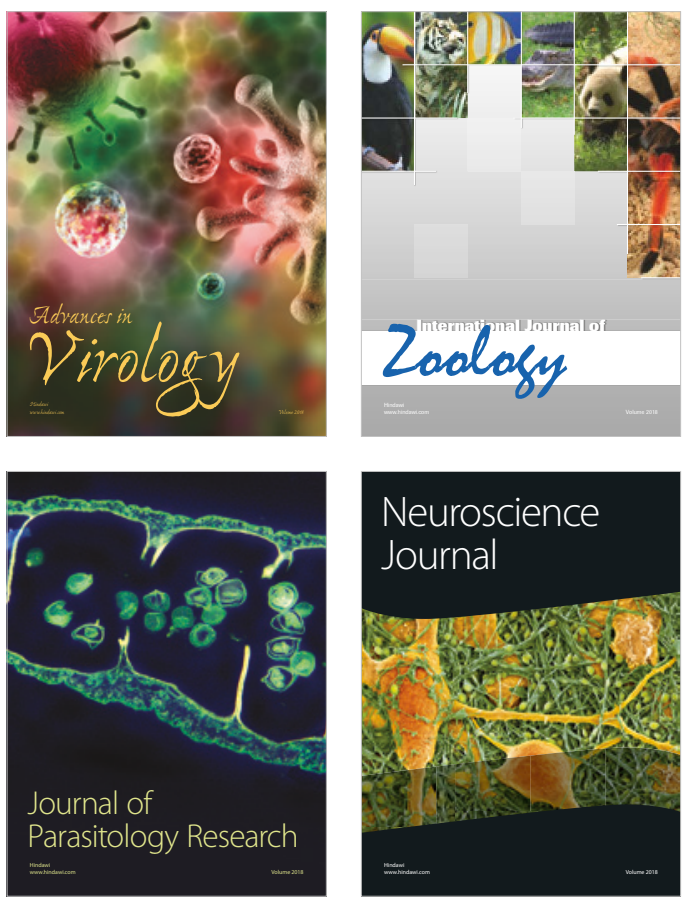
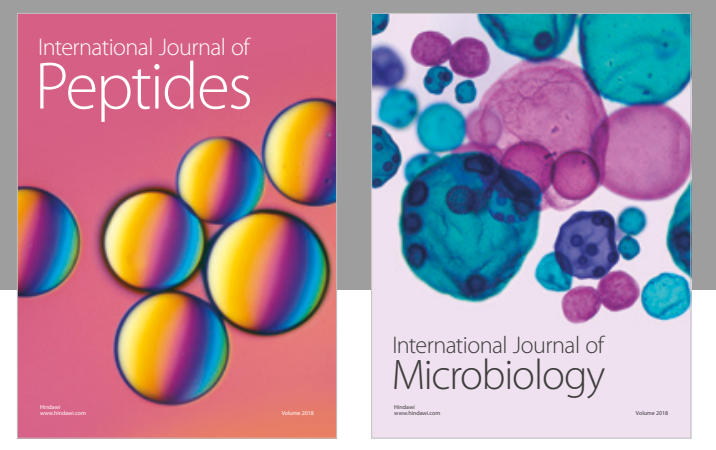

nternational Journal of Microbiology
Journal of
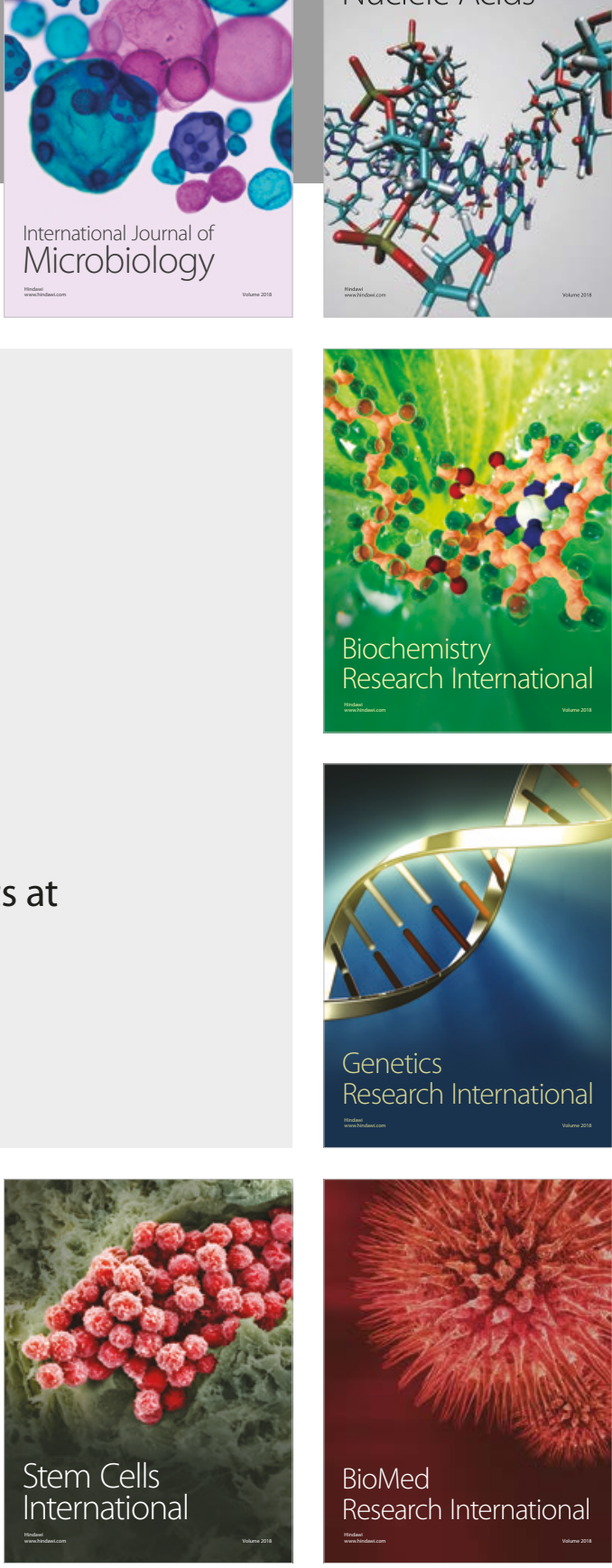
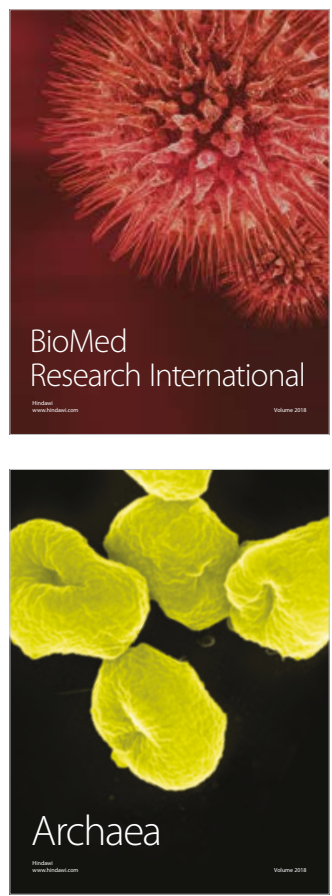